\title{
Dual role of nanoparticles as drug carrier and drug
}

\author{
Hirak Kumar Patra • Anjan Kr. Dasgupta • \\ Sounik Sarkar • Indranil Biswas • \\ Arnab Chattopadhyay
}

Received: 2 November 2010 / Accepted: 7 December 2010/Published online: 1 January 2011

(C) Springer-Verlag 2010

\begin{abstract}
The conventional chemotherapeutic agents used in the treatment of human malignancies are directed nonspecifically against both malignant and nonmalignant cells, often limiting their efficacy with having serious side effects. Recent development of drug delivery vehicles has opened up the possibility of targeted drug delivery systems with the potential of achieving maximum efficacy with minimal toxicity. The possibility of using a nanomaterial as a combinational drug component is intuitively evident as it would compensate the toxicity level by enhancing drug delivery efficiency. Additionally, cell-specific cytotoxicity (reported earlier by our group) of the nanovehicle itself may potentiate a more effective targeted cell killing. In this paper, we explore the possibility of using gold nanoparticles playing the dual role of an anticancer agent and a carrier of a chemotherapeutic drug. This is demonstrated using vincristine sulfate (VS), salt of an alkaloid often used in the treatment of multiple myeloma (MM), and U266 as a test MM cell line. The drug VS shows the expected G2-Mphase arrest of cells. Notably, bare gold nanoparticle shows
\end{abstract}

Electronic supplementary material The online version of this article (doi:10.1007/s12645-010-0011-3) contains supplementary material, which is available to authorized users.

H. K. Patra $\cdot$ A. K. Dasgupta $(\varangle) \cdot$ S. Sarkar

Department of Biochemistry, University of Calcutta,

35 Ballygunge Circular Road,

Kolkata 700019, India

e-mail: adgcal@gmail.com

A. K. Dasgupta

e-mail: adbioc@caluniv.ac.in

S. Sarkar · I. Biswas · A. Chattopadhyay

Institute of Haematology and Transfusion Medicine,

MCH Building (2nd floor), Medical College 88 College Street,

Kolkata 700073, India arrest of the S phase cells that may be particularly important in case of slow-growing malignancies like MM where most of the cells remain in G1 phase of the cell cycle. The VS conjugated gold retains the activity of both gold nanoparticle and VS leading to a synergistic rise of the apoptotic cell population.

Keywords Gold nanoparticle $\cdot$ Vincristine sulfate $\cdot$ Multiple myeloma $\cdot$ Cell cycle $\cdot$ Drug delivery

\section{Introduction}

One important aspect in nanomedicine is focused towards nanoparticle/drug combination. A recent study (Murphy et al. 2008) shows that lipid-cholesterol-based nanoparticle that is targeted towards $\alpha v \beta 3$ integrin (an internal receptor of a number of viruses) can work in conjugation with the chemotherapy drug doxorubicin. The greatest advantage in the nanoparticle based drug delivery system is that the drug can remain active at a significantly lower dose (Barbu et al. 2009; Zhao et al. 2009).

Like integrin, cell cycle has been an important target for new drug development in cancer therapy (de Carcer et al. 2007; Johansson and Persson 2008; Dickson and Schwartz 2009; Raucher et al. 2009). The reason for this target identification is that the agents perturbing the cell cycle are not disease specific and can be exploited in different classes of malignancies. As cell cycle is a cellular network-based event, the cycle can be targeted through multiple routes. The antimicrotubule drug vincristine sulfate (VS), salt of a naturally occurring alkaloid, disrupts microtubules and mitotic spindle, resulting in cell-cycle mitotic arrest at metaphase and apoptosis of cells (Sanchez et al. 2000). VS is one of the most important drugs used in the treatment of 
hematological malignancies as a part of various combination chemotherapy regimens. It is highly lipophilic, and therefore attains a several-hundred-fold higher intracellular than extracellular concentration on administration. However, over expression of the multidrug resistance (Huet et al. 1998; Legrand et al. 1999) transporters P-glycoprotein (PGP) or multidrug resistance-associated protein (MRP) can reduce the intracellular accumulation leading to drug resistance (Calatozzolo et al. 2005; Rauch and Pluen 2007; $\mathrm{Ma}$ et al. 2009). Like any chemotherapy drug, VS is also not free from side effects, its dose limiting toxic effect being neurotoxicity. Multiple myeloma (MM) is a differentiated clonal B cell tumor usually comprising slowly proliferating plasma cells (Zhao et al. 2010a, b). MM constitutes around $1 \%$ of all malignant diseases and $10 \%$ of hematological malignancies. MM is a relatively slowgrowing malignancy and is therefore difficult to treat (Dvorak 2006). Most myeloma cells are in the G1 phase of the cell cycle and are hence poor targets for chemotherapy because they have ample time to repair any DNA damage caused by many of the chemotherapeutic drugs before they proceed to the next cell division. VS, being a drug, causing metaphase arrest, form an important component of various antimyeloma combination regimens.

Extending our previous example of NP-based drug delivery systems, we can ask whether it is possible to design a novel NP-based delivery systems that would accentuate the activity of a drug, like VS at lower concentration. While lowering of concentration should lead to minimization of the observed side effects, it is also worth exploring whether it is possible to impart higher efficiency in chemotherapy by arresting the cell at an earlier phase of cell cycle. The direct effect of nanoparticle itself on cell cycle would enhance the chemotherapeutic potential of the drug.

Direct cancer cell killing by gold nanoparticles (GNP) was proposed earlier by our group (Patra et al. 2007) is different from the laser-mediated thermolysis (Lapotko et al. 2006) using GNP. Two disadvantages of the latter approach are the relative inaccessibility of the laser to the target site, and thermal injury induced by the incident laser on nontarget cell confined nanosurface. Recently, Rotello's group has shown that nanosurface toxicity is indeed possible and the toxicity largely depends upon hydrophobicity of the nanosurface (Daniel et al. 2010). Although Rotello's work was not aimed for any anticancer drug formulation and it was aimed primarily to evaluate the toxicity of gold nanoparticle, we found the report contextual, as in our view the 'toxicity' is a contextual theme whose chemical or biochemical imprint can have differential realization depending on the study system. The important message from this and other works done by Rotello and his group (Chompoosor et al. 2010;
Daniel et al. 2010; Zhu et al. 2010), is that nanosurfaceinduced ROS generation and subsequent DNA damage may give the nanosurface a special role as a drug. This finding inspired us to attempt a nanoformulation in which both the activity of the nanosurface and that of the drug can be combined and the drug delivery problem is also addressed.

\section{Materials and methodology}

\subsection{Preparation of gold nanoparticle}

The gold nanoparticles were prepared by the standard method as described by Turkevich and Frens with some modifications (Kimling et al. 2006). In brief, an aqueous solution of $\mathrm{HAuCl}_{4}(250 \mu \mathrm{M}, 25 \mathrm{~mL})$ was brought to near boiling condition upto $100^{\circ} \mathrm{C}$ and stirred continuously with a magnetic stirrer. Freshly prepared trisodium citrate solution $(600 \mu \mathrm{M}$ final concentration) was added quickly, resulting in a change in the solution color from pale yellow to deep red. After obtaining a stable deep red color, the solution was then allowed to cool down to room temperature with continuous stirring.

\subsection{Preparation of gold nanoparticle VS conjugate}

Freshly prepared aqueous solution of VS was prepared at a stock concentration of $10 \mu \mathrm{g} / \mathrm{ml}$. Aqueous solution of $\mathrm{HAuCl}_{4}(250 \mu \mathrm{M}, 25 \mathrm{~mL})$ was heated to $60^{\circ} \mathrm{C}$ and stirred continuously with magnetic stirrer. Two working solutions of VS namely, GNP-VS1 and GNP-VS2 at final concentrations of $0.5 \mu \mathrm{g} / \mathrm{ml}$ for GNP-VS1 and $1.0 \mu \mathrm{g} / \mathrm{ml}$ were prepared, respectively. They were then added to the warm auric chloride solution with stirring for $10 \mathrm{~min}$. Trisodium citrate solution $(600 \mu \mathrm{M}$ final concentration) was added quickly and the temperature was increased to near boiling without allowing the solution to boil, resulting in a change in solution color from pale yellow to bluish red. After obtaining a stable color, the solution was then allowed to cool down to room temperature with continuous stirring.

\subsection{Characterization of the nanoparticles}

The hydrodynamic diameter of the GNP and its conjugate with VS were measured using Malvern NanoZS80 (Malvern, UK). The laser Doppler velocitimetry module of the same instrument was used to measure the zeta potential of the nanoparticle. This technique efficiently measures the relative velocity of tiny particles with respect to the fluid. The zeta potential is purely an electro kinetic property of the electrical double layer surrounding the subject but not 
the surface of the subject itself. The conjugation was further characterized using Plasmon resonance of the GNP and its conjugates with VS using Thermo UVVisible Spectrophotometer.

\subsection{Cell culture}

MM cell line U266 was procured from NCCS, Pune, India and was maintained in DMEM (Gibco-BRL, NY) supplemented with $10 \%$ heat-inactivated fetal bovine serum (Gibco-BRL, USA), $5 \times 10^{-5} \mathrm{M}$ 2-ME, $10 \mathrm{mM}$ Hepes, $2 \mathrm{mM}$ L-glutamine, $100 \mathrm{U} / \mathrm{ml}$ penicillin, $100 \mu \mathrm{g} / \mathrm{ml}$ streptomycin, and $1 \mathrm{mM}$ sodium pyruvate. The cell line was terated with nanoparticle concentration $(125 \mu \mathrm{M})$ and by VS at a concentration that equivalent to that in GNP-VS2 conjugates. All experiments on the treated cell line were done after $72 \mathrm{~h}$.

\subsection{MTT assay}

In vitro cytotoxicity of GNP and its drug conjugate on MM cells were determined by measuring 3-[4,5-dimethylthiazol2-yl]-2,5-diphenyltetrazolium bromide (MTT) dye absorbance of living cells. In brief, MM cells $\left(5 \times 10^{6}\right.$ cells per assay) were grown in tissuce culture plates (NUNC, USA). After treatment with GNP and GNP-VS for $48 \mathrm{~h}, 20 \mu \mathrm{L}$ MTT (Sigma, USA) solution ( $5 \mathrm{mg} / \mathrm{mL}$ in PBS) was added to each plate and were incubated for additional $4 \mathrm{~h}$ at $37^{\circ} \mathrm{C}$. Following this, the MTT solution was decanted off and the formazan crystals formed in the viable cells were dissolved with $200 \mu \mathrm{L}$ DMSO. The absorbance was taken at $570 \mathrm{~nm}$ keeping the reference at $405 \mathrm{~nm}$ to measure the level of response to GNP and GNP-VS.

\subsection{DNA fragmentation assay}

The DNA fragmentation was measured after extraction of DNA from a constant number of cells (approximately $1 \times$ $10^{6}$ ). In brief, treated and control cells were harvested, pelleted at 1,600 rpm for $5 \mathrm{~min}$, washed twice with PBS, and resuspended in $60 \mu \mathrm{L}$ of lysis buffer $(10 \mathrm{mM}$ EDTA, $50 \mathrm{mM}$ Tris- $\mathrm{HCl}, \mathrm{pH}$ 8.0, $0.5 \%$ SDS, $0.5 \mathrm{mg} / \mathrm{mL}$ proteinase-K). The solution was mixed and incubated overnight at $37^{\circ} \mathrm{C}$ followed by addition of $3 \mu \mathrm{L}$ of $10 \mathrm{mg} / \mathrm{mL}$ DNAse-free RNase-A. After digestion of RNA, gel electrophoresis was performed on a $1.5 \%$ agarose containing ethidium bromide. The gels were documented by imaging with UV transillumination.

\section{Cell-cycle analysis}

Cell-cycle assay for the amount of DNA distribution was determined by staining DNA with PI (propidium iodide)
(Sigma). In brief, after treatment for $24 \mathrm{~h}$, nearly $1 \times 10^{6}$ cells were washed in PBS and fixed in $70 \%$ ethanol and kept for overnight at $-20^{\circ} \mathrm{C}$. The fixed cells were washed twice with PBS and then subjected to PI $(10 \mu \mathrm{g})$ and RNase treatment at $37^{\circ} \mathrm{C}$ for $1 \mathrm{~h}$. Cell-cycle analysis by flow cytometry was done to determine the percentage of cells in different phases of cell cycle using a FACS Calibur (BD Biosciences, USA) instrument.

\section{Annexin V staining}

The annexin $\mathrm{V}$ staining was performed for the determination and quantitation of the percentage of cells undergoing apoptosis for GNP and its drug conjugate (GNP-VS). In brief, after treatment, the cells were washed twice with cold PBS and resuspended in binding buffer $(10 \mathrm{mM}$ HEPES/NaOH, pH 7.4, $140 \mathrm{mM} \mathrm{NaCl}$, $2.5 \mathrm{mM} \mathrm{CaCl}_{2}$ ) at a concentration of $1 \times 10^{6}$ cells $/ \mathrm{mL}$. A $100 \mu \mathrm{L}$ of the suspended cells in binding buffer was transferred to a $5-\mathrm{mL}$ culture tube, and $5 \mu \mathrm{L}$ of Annexin V-FITC and $10 \mu \mathrm{L}$ of PI were added. The solution was mixed properly and incubated for $15 \mathrm{~min}$ at room temperature in the dark. At the end of incubation, $400 \mu \mathrm{L}$ of binding buffer was added, and the cells were analyzed immediately by flow cytometry. Flow cytometric analysis was performed with the FACSCalibur using the CellQuest software (BD, USA).

\section{Results}

\subsection{GNP and its conjugates with VS}

\subsubsection{UV-VIS spectroscopy}

Figure 1 describes the normalized UV-VIS absorption spectra of GNP (blue line) and its conjugates, GNP-VS1 (green line) and GNP-VS2 (red line). An intense and sharp surface plasmon absorption peak at $517 \mathrm{~nm}$ attributed to GNP collective electron oscillations or localized surface plasmon resonance is presented and is a feature of gold nanoclusters. It is clear that the plasmon absorption peak of GNP shifts to $519 \mathrm{~nm}$ (GNP-VS1) and 523(GNP-VS2) nm after the conjugation with VS. The resonance wavelength and bandwidth of nanoparticles are dependent on the particle size and shape, the refractive index of the surrounding medium and the temperature.

\subsubsection{Dynamic light scattering}

Dynamic Light Scattering study shows (Fig. 2a) statistical size distribution of GNP and its drug conjugates GNP- 
Fig. 1 The normalized UV-VIS absorbance spectra of GNP and GNP-VS. The $\lambda \max$ is 517,519 , and $523 \mathrm{~nm}$ for GNP, GNPVS1, and GNP-VS2, respectively

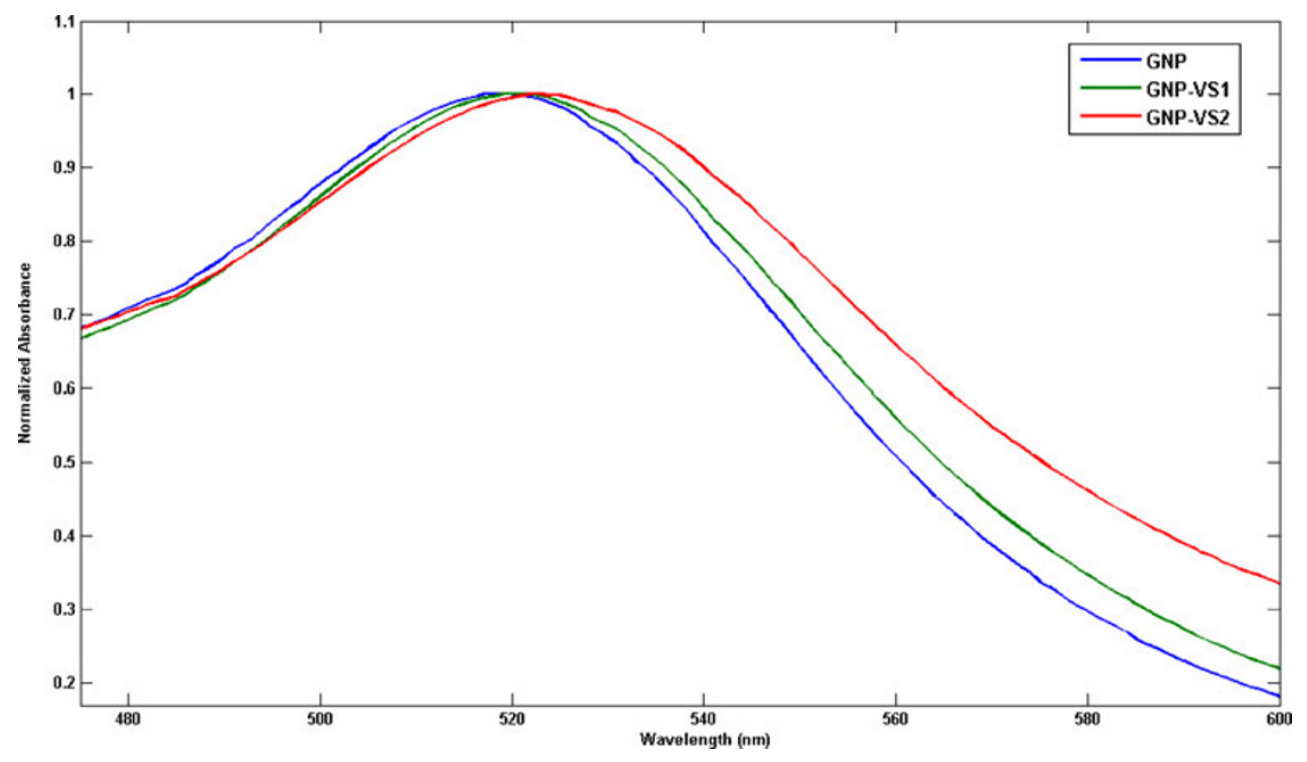

VS1 and GNP-VS2. The hydrodynamic sizes of the nonmaterial are around $25 \mathrm{~nm}$. The comparative distribution (see Fig. S2 in the Electronic supplementary material) shows the broadening of the distribution pattern that can be described due to the formation of the GNP-VS (green and blue) than that of the GNP (red). From the measurement of the zeta potential $(\varsigma)$ data, it has been shown (Fig. 2b) there is a gradual shift of the $\varsigma$ value when the GNP has been conjugated with VS. This evidence is expected because the drug molecules will replace the citrate molecules during conjugation as a result of which there is a gradual drop and shift of $\varsigma$ value. In this case the steric factor of the drug molecule will contribute to stabilize the particle in colloid.

\subsubsection{Fourier transform infrared spectroscopy}

The Fourier transform infrared (FTIR) spectra of VS, GNP (see Electronic supplementary material) and GNPVS conjugates with VS are shown in Fig. 3. The peaks are characterized and marked. Direct evidences of conjugation was observed from peaks at $1,910-1,970 \mathrm{~cm}^{-1}$ for $\mathrm{C}-$ $\mathrm{H}$ alkyl stretching, $2,549 \mathrm{~cm}^{-1}$ due to $\mathrm{S}-\mathrm{H}$ deformation and $701 \mathrm{~cm}^{-1}$ for thiol stretching. $\mathrm{C}=\mathrm{O}$ stretch at $1923 \mathrm{~cm}^{-1}$ and $1,666 \mathrm{~cm}^{-1}$ along with the $\mathrm{N}-\mathrm{C}$ stretch at $1,621 \mathrm{~cm}^{-1}, 1,615 \mathrm{~cm}^{-1}$ confirms the bond level interaction of VS with GNP. Several other peaks appeared at 758 and $1,620 \mathrm{~cm}^{-1}$, which are associated with the $\mathrm{N}-$ $\mathrm{H}$ deformation and stretching vibration, respectively. These results suggest the conjugation of VS with GNP via bond between gold and sulfur, gold and nitrogen in the stable drug-gold conjugate. For further analysis and details of FTIR see Fig. S2 in the Electronic supplementary material.

\subsection{Anticancer activity}

\subsubsection{MTT assay}

MTT (3-(4,5-dimethylthiazol-2-yl)-2,5-diphenyltetrazolium bromide) has been employed to study the survival rate of the cells in Fig. 4a. Notably, both VS- and GNP-treated cells showed lower percentage of survival. Further reduction in survival was observed in GNP-VS1 and GNP-VS2 cells with lesser survival with GNP_VS2.

In contrast to the cancer cells, PBMC (peripheral blood mononuclear cells) did not show any alteration in percentage survival pattern when challenged with GNP, VS, GNPVS conjugates (Fig. 4b).

\subsubsection{PI uptake}

Cell damage was also assayed with propidium iodide (PI) uptake studies (Fig. 5). Plasma membrane integrity was altered in presence of VS, GNP, and GNP-VS conjugates. In each case, there was higher density of points (representing individual cell) in the upper half of the dot plot (Fig. 5a) implying higher PI entry after treatment with VS, GNP, and GNP-VS conjugates, the effect being dramatically higher for GNP-VS2.

Figure 4 describes survival rate on the basis of the metabolic activity of the cells. The reducing nature in GNPVS conjugates seems to retard the metabolic rate of the population. This is illustrated by the relative bar heights in Fig. 4. Importantly, there is also an associated loss of plasma membrane integrity upon such conjugation causing enhancement of PI uptake. Reverse is the case for intact plasma membranes, where PI's are excluded even in case of cell death (see Fig. 5). 
Fig. 2 a Intensity statistics of hydrodynamic diameter of the nanoparticles is described. $\mathbf{b}$ Zeta potential of nanoparticles-GNP (red line) is around $-40 \mathrm{mV}$ and gradually shifted to $-20 \mathrm{mV}$ during conjugation with VS a

Statistical Distribution of Size

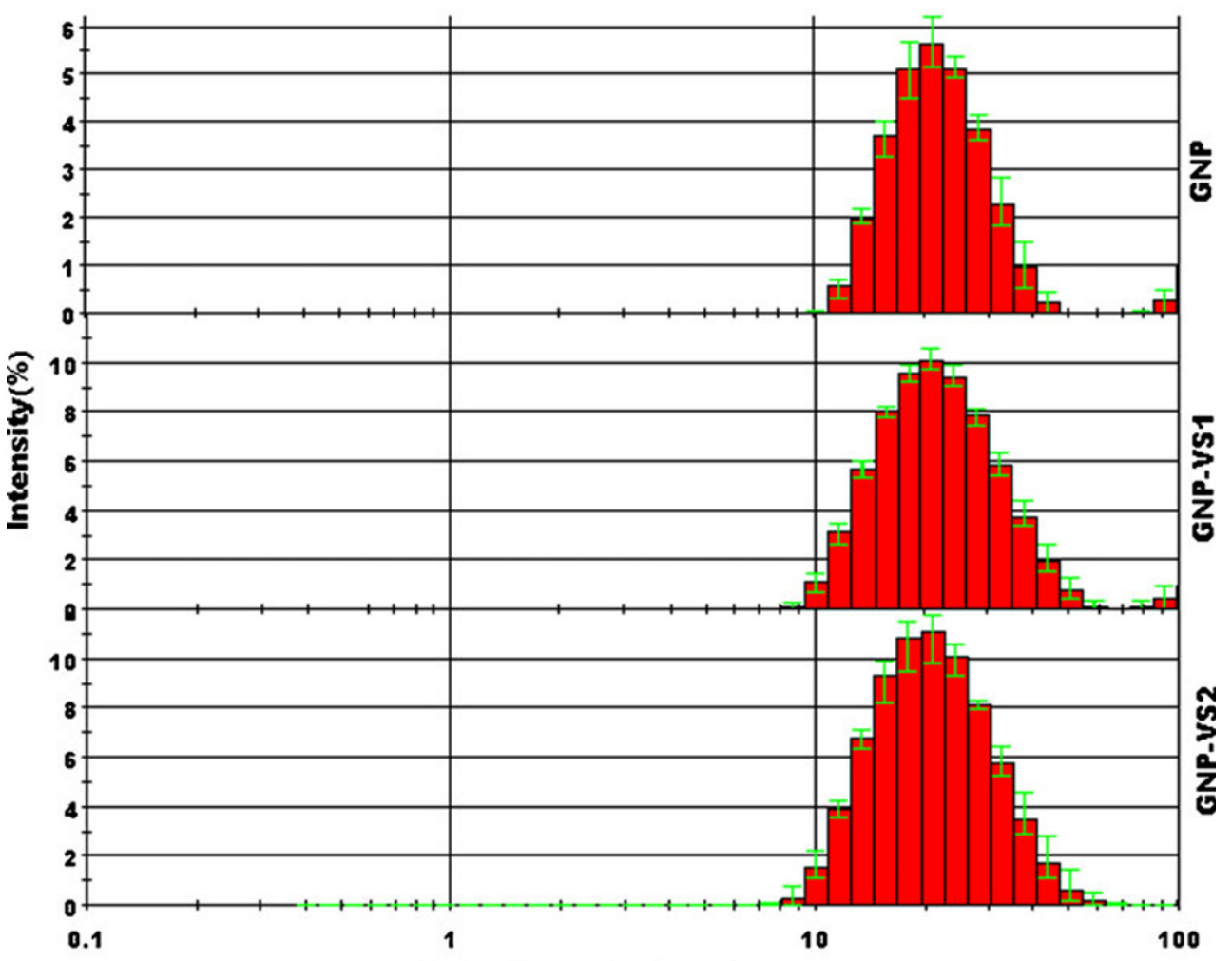

Hydrodynamic Diameter (nm)

b

Zeta Potential Distribution

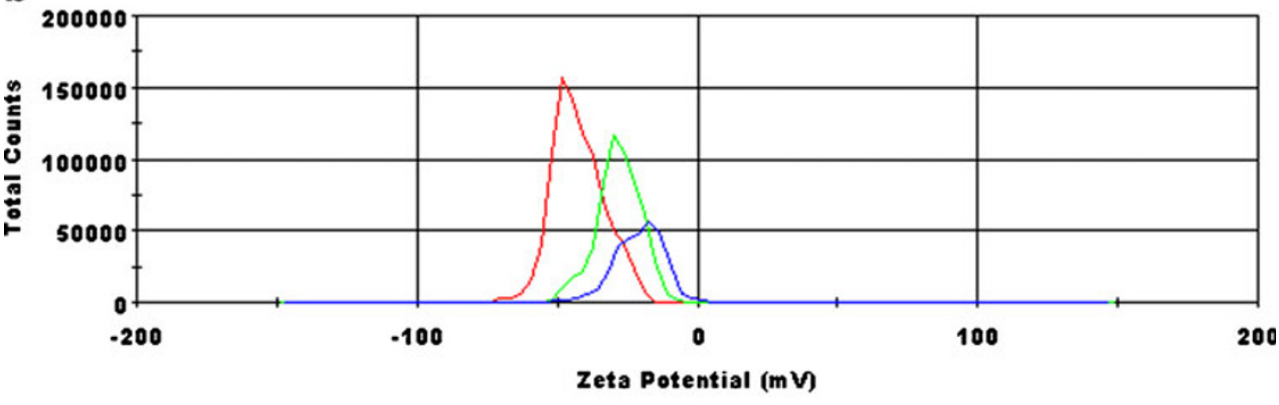

\subsubsection{Annexin- $V$}

We now need to explore whether the observed cell death is mediated by apoptosis or necrosis, the two different mechanisms by which cells die (Lieberthal et al. 1996; Kostrzewa 2000; Anselmi et al. 2002; Catelas et al. 2005; Vairetti et al. 2005). The dynamics in these two processes differ greatly. The plasma membrane damage occurs as a primary event during necrosis whereas a delayed but massive programmed phenomenon occurs during apoptosis. In Fig. 5, we have shown only the population of the dead cells, but the mechanism of the event is unexplored. In apoptotic cells, the membrane phospholipid phosphatidylserine is flipped from the inner to the outer leaflet of the plasma membrane, hence exposed to the extracellular environment (Kekre et al. 2005). Annexin V, Ca2+-dependent phospholipid-binding protein, conjugated with FITC has been used with flow cytometry to measure this event. Annexin V-FITC assay as shown in Fig. 6 has identified apoptosis as the early stage event. Interestingly, this followed the DNA fragmentation in the nucleus which is a late apoptotic event (data not shown). The population density as shown in Fig. 6a gradually shifted to higher fluorescence while conjugated with VS. The qualitative shifting of the population has been shown in Fig. $6 \mathrm{~b}$ and the respective quantitation has been shown in Fig. 6c. Now Fig. 6 describes that the cells are undergoing apoptosis in presence of either GNP or VS. For GNP conjugated with VS there is a cumulative effect. 


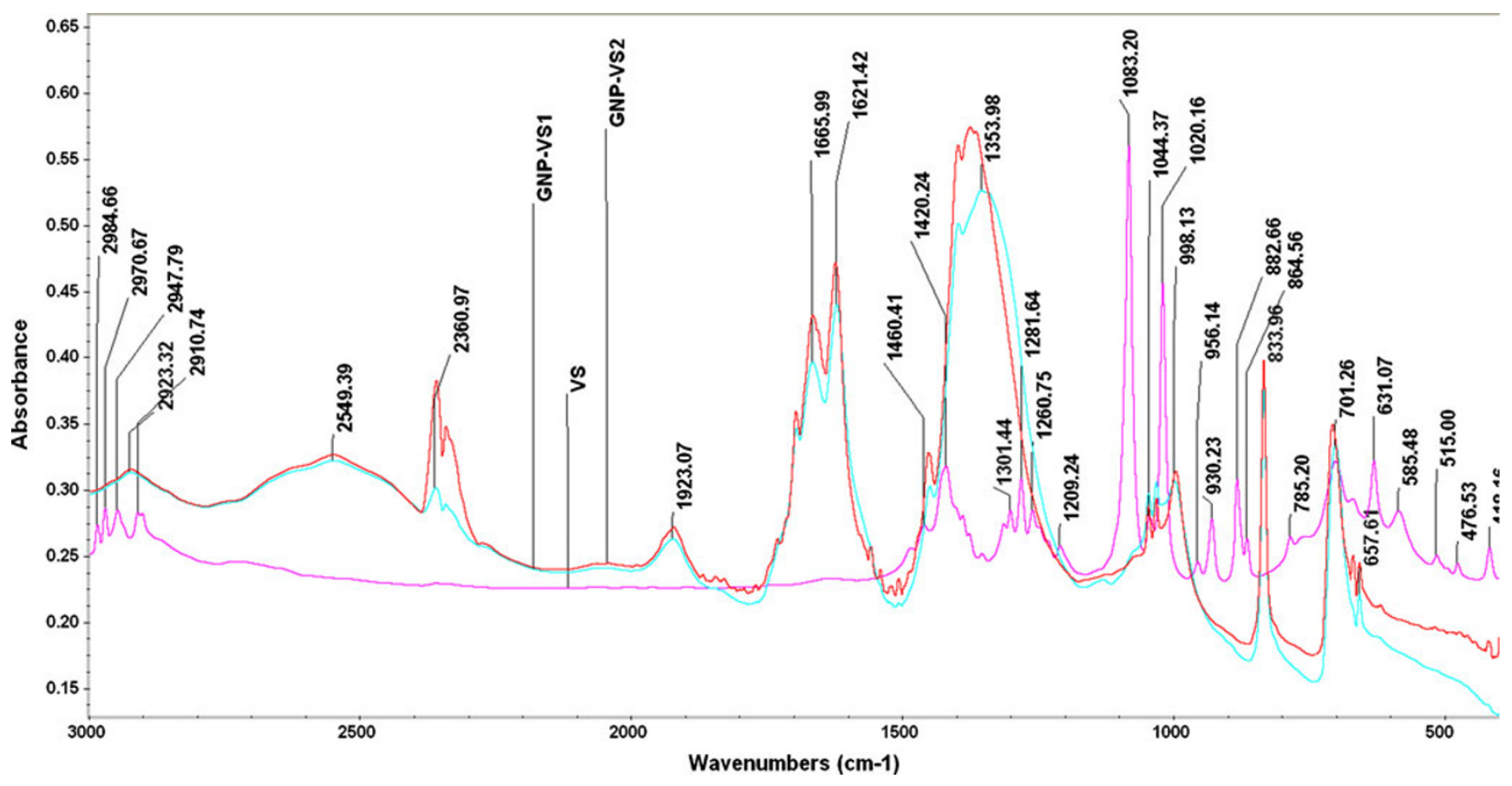

Fig. 3 The Fourier transform infrared spectrum of VS (pink), GNP-VS1 (green) and GNP-VS2 (red), respectively. The alteration in the peak intensity and pattern was marked with corresponding wavenumbers $\left(\mathrm{cm}^{-1}\right)$

Dual Staining of annexin V-FITC with propidium iodide (PI) was then used (Fig. 7) for identification of early apoptotic cells (PI negative, Annexin V-FITC positive) from dead cells (PI positive, annexin V-FITC positive). In Fig. 7, the different kinetics of Propidium iodide incorporation by necrotic and apoptotic cells (annexin V positive) after treatment with different GNP and its VS conjugates have been represented along with the control. Figure $7 \mathrm{a}$ represents the basis for the flow cytometric identification (dot plot) of different cellular subsets by defining appropriate quadrant. The dual positivity (both annexin $\mathrm{V}$ positive and Propidium Iodide positive) of the GNP-VStreated cells strongly implies that the cells are undergoing apoptosis.

\subsubsection{Cell cycle}

As VS-mediated chemotherapy is primarily targeted towards the cell cycle, cell-cycle analysis was performed with GNP, VS, and GNP-VS. The relative cellular DNA content was identified by the distribution of the cells during the various phases of the cell cycle. Four distinct phases were recognizing in a proliferating cell population as represented in Fig. 8: G0/G1, S (DNA synthesis phase), and G2/M-phase (mitosis), indicated as M2, M3, and M4, respectively, in the histogram. In G2 and Mphase there is a single population of cells showing identical DNA content (M4). VS was found to affect cells in interphase, producing a transient G2 block with
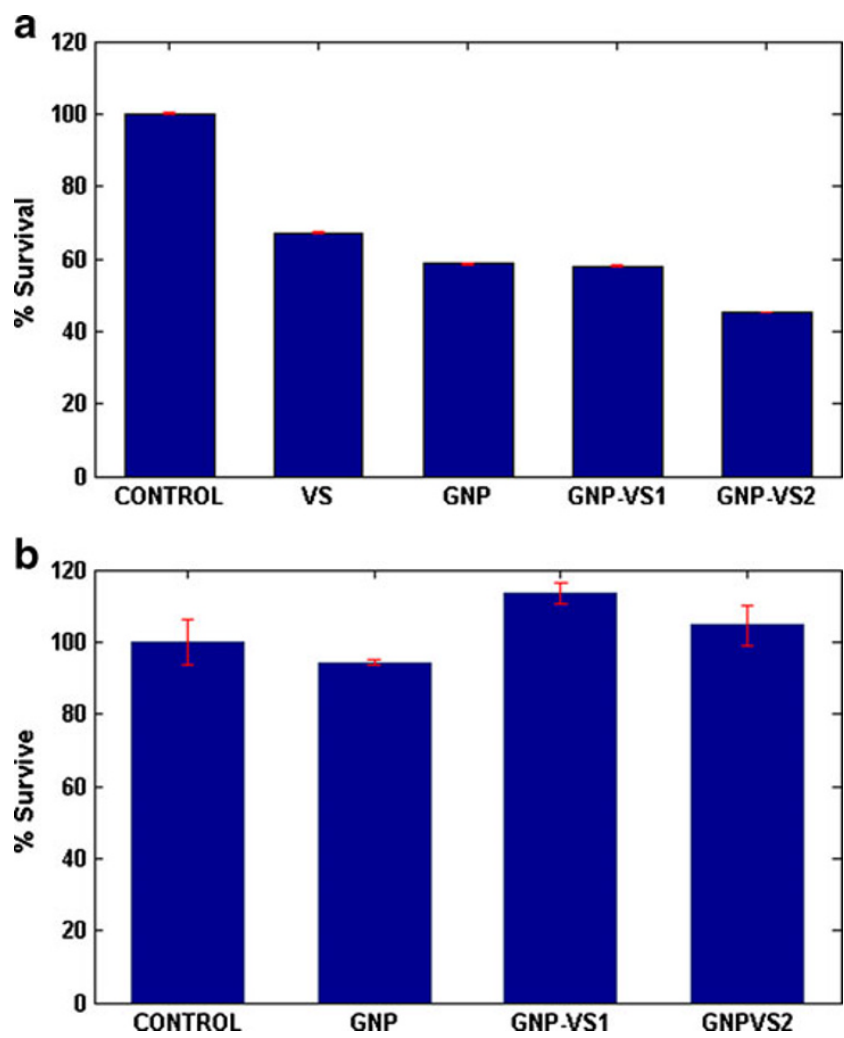

Fig. 4 a The survivality of U266 cell line with corresponding control and treatment. b The corresponding response to PBMC 

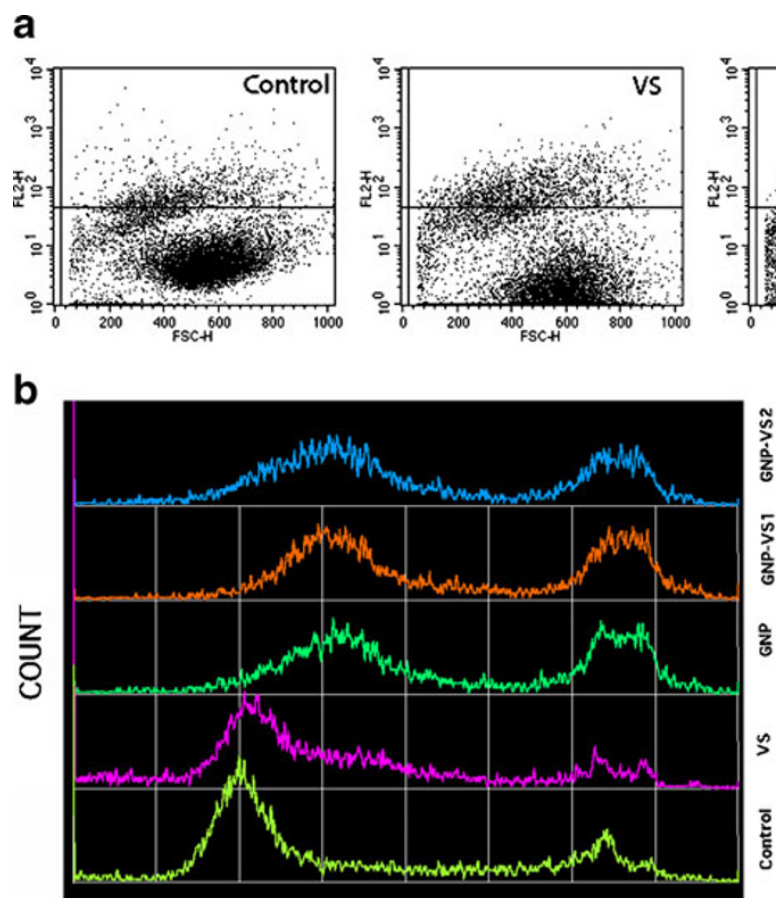

PI INTENSITY INDEX

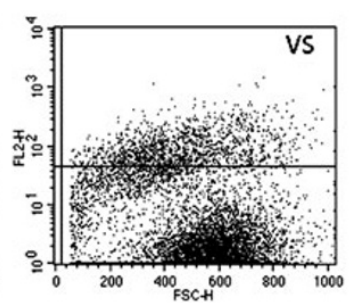

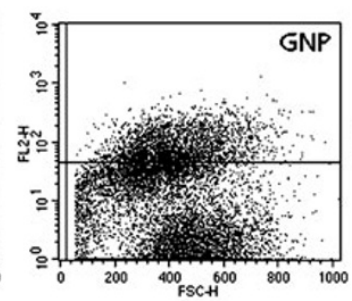
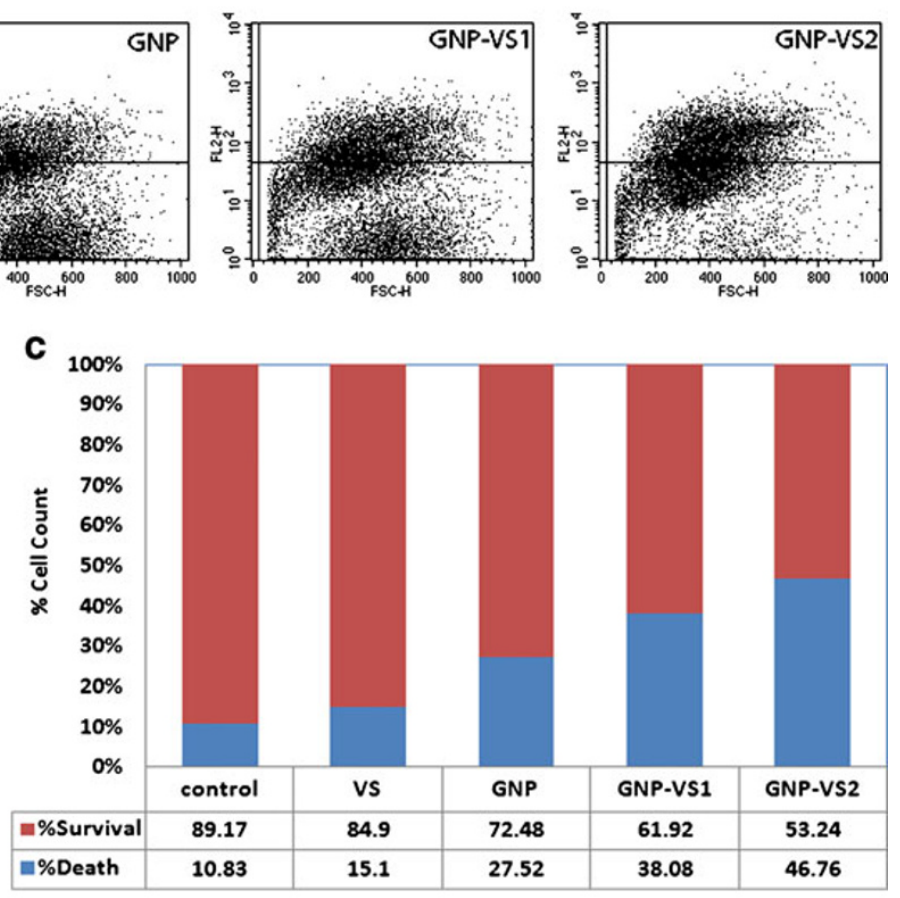

Fig. 5 a The dot plot of PI uptake of the myeloma cell line U266. b The histogram pattern of the population with PI uptake. c The quantitative estimation of the PI uptake

all drug conjugates (GNP-VS1 and GNP-VS2). Metaphase index in case of VS was increased as expected, vincristine being a well-known G2/M blocker.

The cell-cycle pattern was affected by GNP, and in a way that was significantly different from cell-cycle shift induced by VS. In case of GNP, there seems to be an arrest at the $\mathrm{S}$ phase of the cell cycle. As shown in Fig. 8a, the G2/M-phase cells are increasing in case of treatment with VS. From the histogram described in Fig. 8a, the GNP-VS conjugates show the cumulative
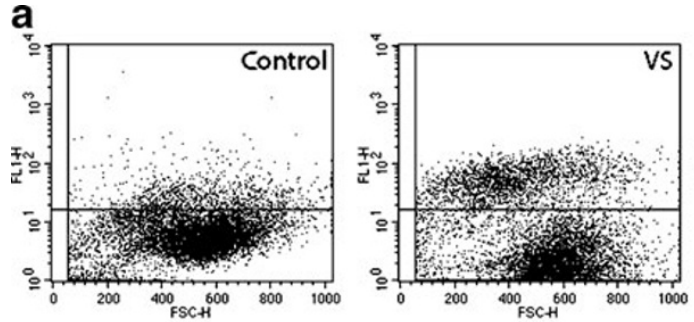

b

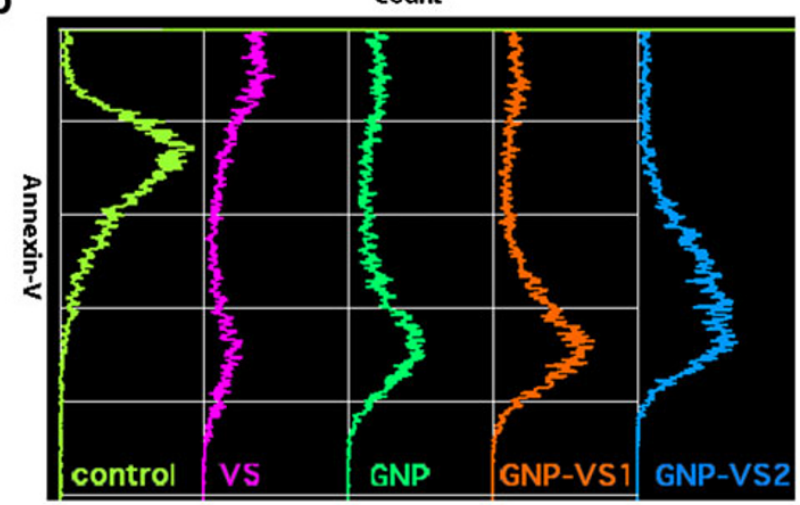

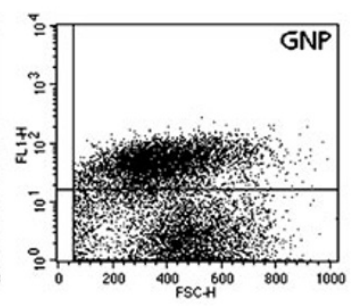
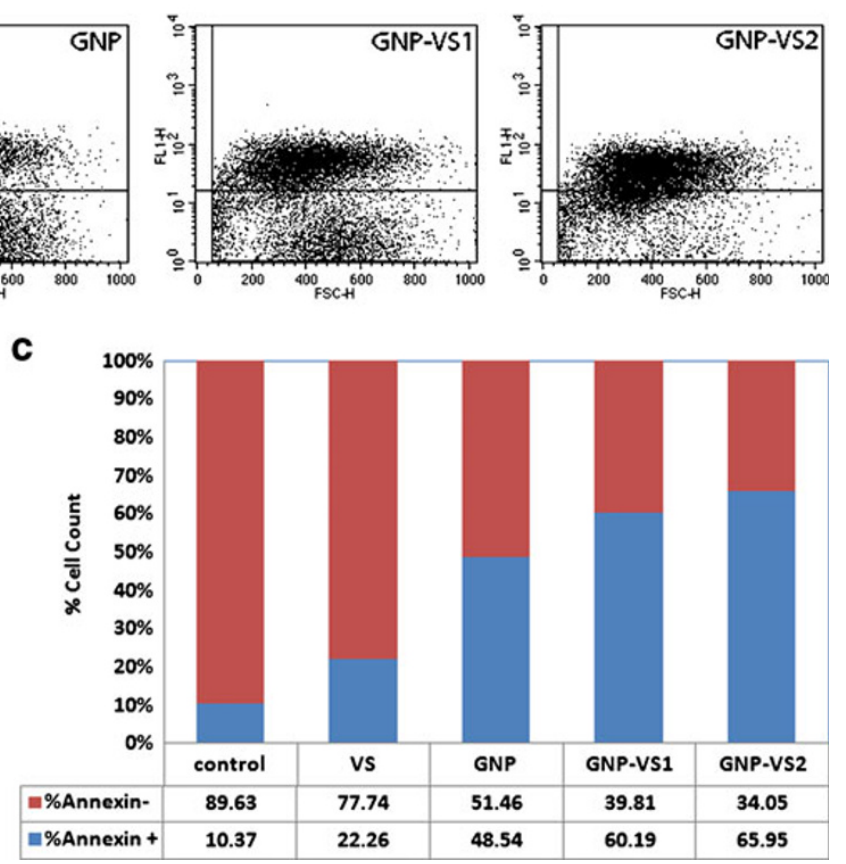

Fig. 6 a The dot plot of the annexin V staining. b The histogram pattern annexin V staining. $\mathbf{c}$ The quantitative estimation of annexin V staining 
response of both the cell-cycle effectors namely GNP and VS. Figure $8 \mathrm{~b}$ illustrates the different response of the cell cycle using a contour plot, in which the VS-, GNP-, and the GNP-VS-induced population shift are clearly classified. The analysis further reveals that there is an increase in less-than- $2 n$ population. This provides evidence for an onset of DNA fragmentation at the late apoptotic phase of cells in presence of VS, GNP, or GNP-VS.

$\mathrm{G} 2 / \mathrm{M}$ population is increased in response to VS as expected, implying a mitotic block, a well-known signature of VS. On the other hand, in case of GNP-VS an additional feature, namely, a decrease of the G0/G1 cell population is thus observed.

Conjugation of drug (here VS) with gold nanoparticle is done successfully by a novel synthesis method. After conjugation, the newly postulated drug (GNP-VS) has achieved unique cell killing properties. The results indicate that Gold conjugated vincristine nanoparticle kills cancer cell line by blocking at synthesis phase (S phase) of cell cycle.

The use of GNP as a drug delivery agent is already established. This is, to our knowledge, the first report describing cell-cycle perturbation by gold nanoparticles. The cumulative effect of GNP and VS on cell cycle makes the combination prospective in chemotherapy of slowgrowing cells like MM. The dual role of GNP as a drug carrier and a drug affecting the cell cycle is also an important outcome of the work.

\section{Discussion}

Killing cells remains the backbone of chemotherapeutic approaches to human malignancies. Most of the conventional chemotherapeutic agents are directed nonspecifically against both malignant and nonmalignant cells, limiting the efficacy of the drugs vis-à-vis enhancing their toxicity. As such, intense research is going on to develop newer agents targeted specifically against malignant cells. VS is the salt of a naturally occurring nitrogenous base derived from the pink periwinkle plant, Catharanthus roseus, vincristine is an asymmetric dimeric compound that binds to the protein tubulin and, at low concentrations, inhibits microtubule dynamics. At higher concentrations, it disrupts microtubules and mitotic spindle, resulting in cell-cycle mitotic arrest at metaphase. A smart formulation of the drug (VS) that further affects the cell cycle without invoking any appreciable toxicity is likely to be welcome in MM treatment. As VS is primarily eliminated through the liver into the bile and feces, patients with obstructive liver disease are more susceptible to its toxic effects, requiring a dose reduction depending upon the serum bilirubin concentration. Vincristine uniquely spares the kidney and the bone marrow. As a result, they are continued to be used extensively in the treatment of various hematological malignancies, as a component of combination chemotherapy regimens that also includes more myelotoxic agents. As

\section{a}
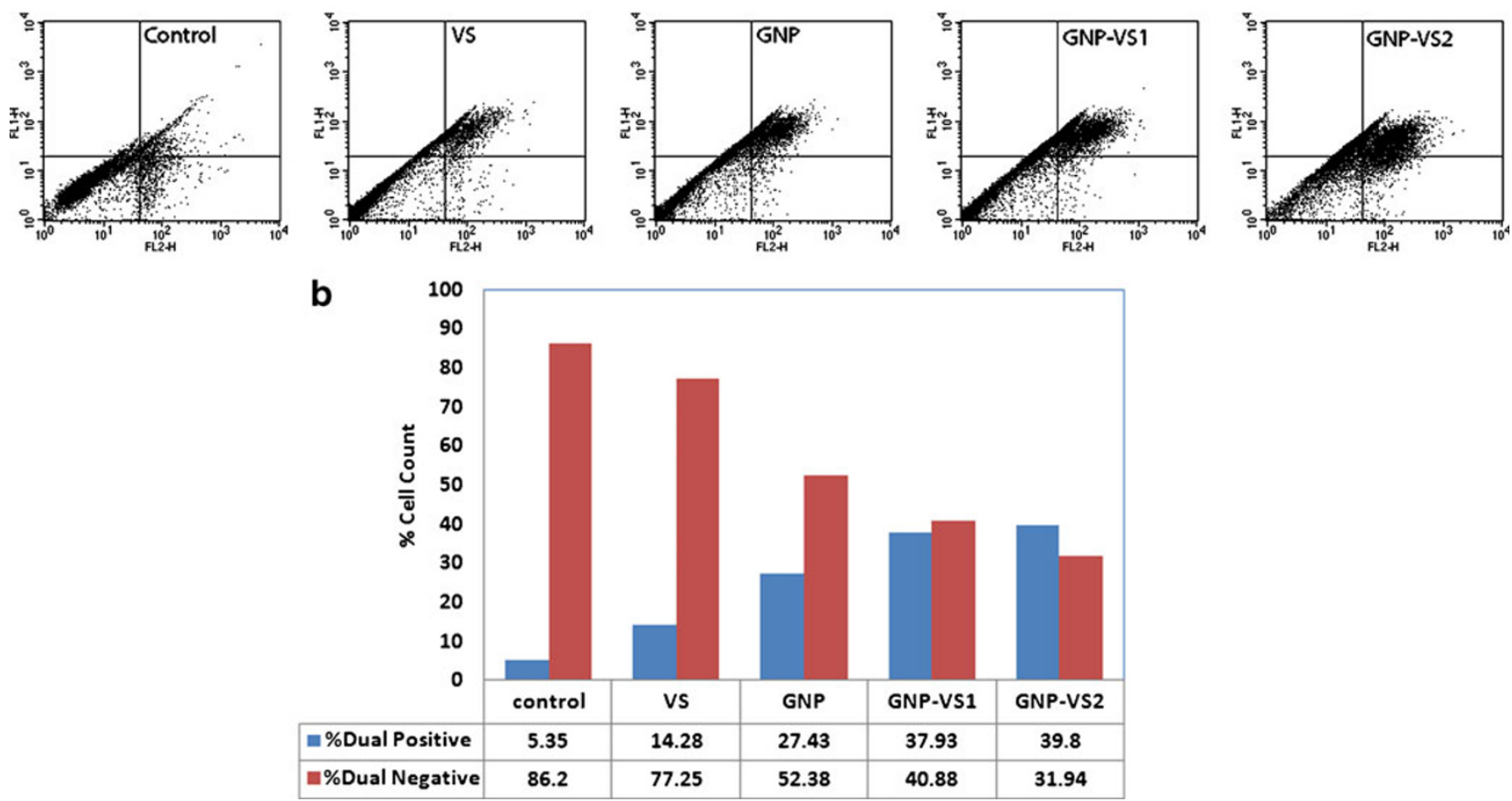

Fig. 7 a The dot plot of dual (annexin V-FITC and PI) staining. b The quantitative estimation of dual staining 
a
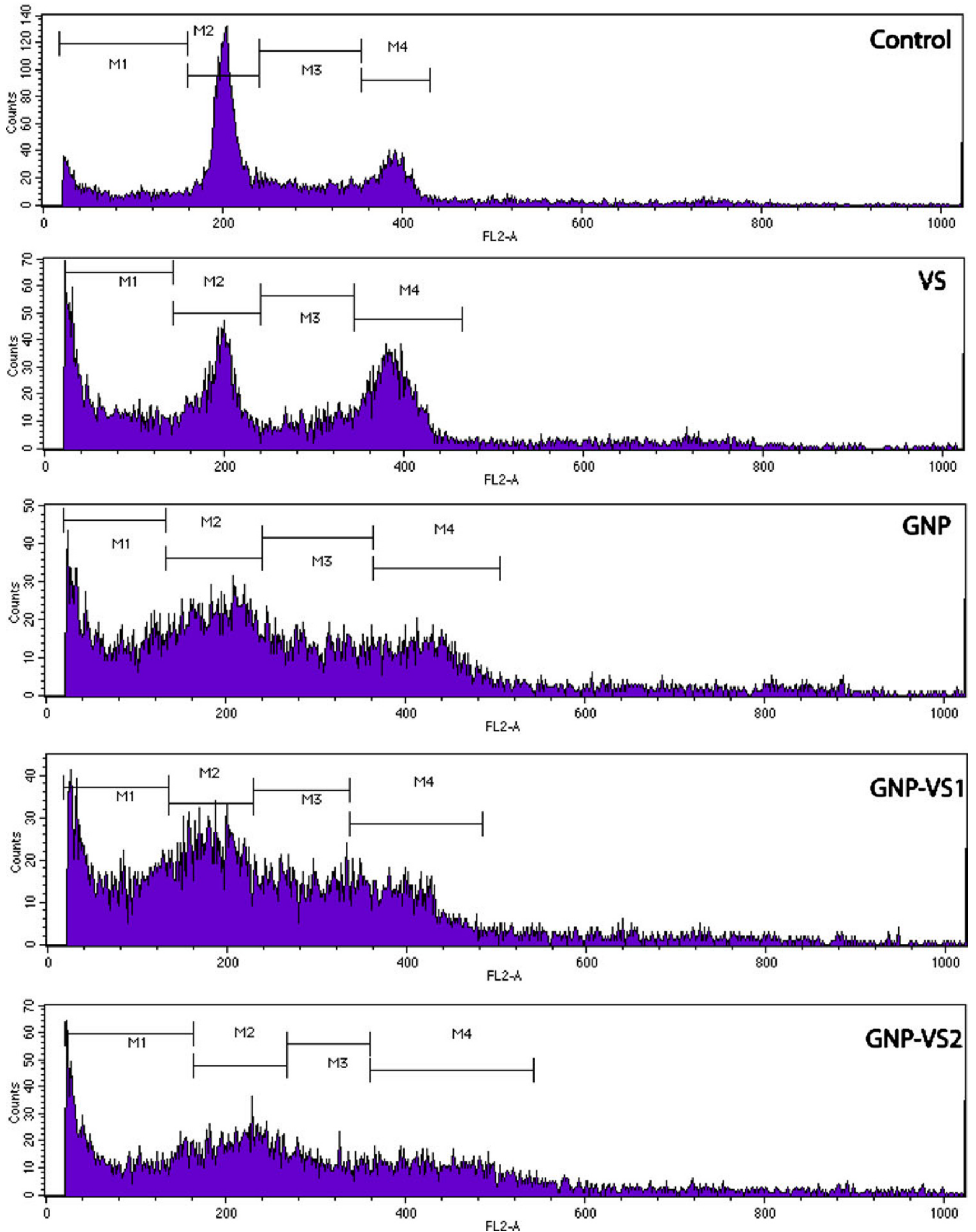

Fig. 8 a Histogram of DNA content of the population. b The contour plot of the DNA content. c-d The quantitative estimation of cell cycle 

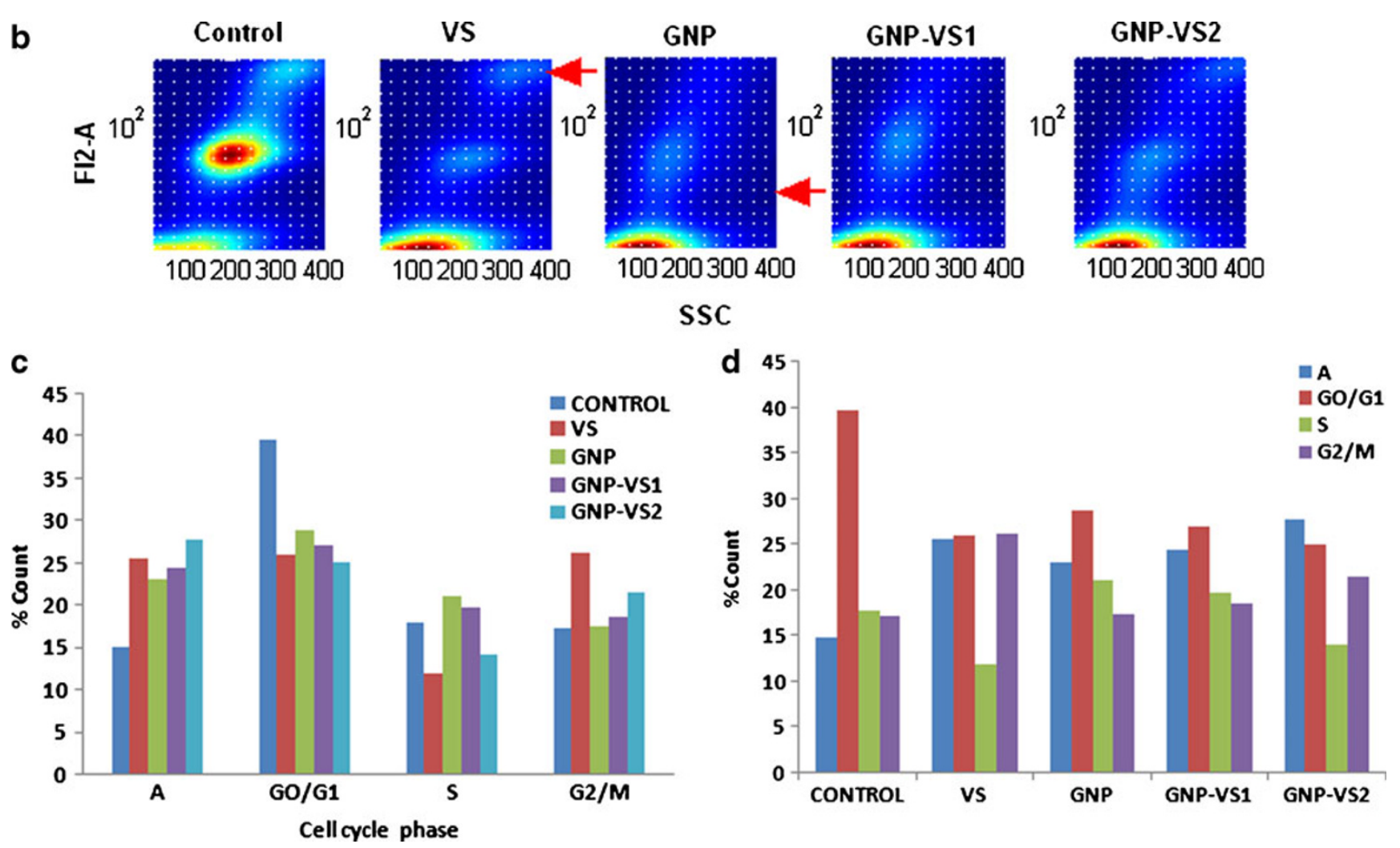

Fig. 8 continued.

nanoparticles are shown to reduce the required VS dose (see Figs. 4 and 5), the GNP based drug formulation is expected to reduce the toxic side effects associated with conventional chemotherapy. The arrest at $\mathrm{S}$ phase cells by GNP may be of potential important to MM therapy. The renal sparing action of VS has helped design chemotherapy regimens, like vincristine-adriamycin-dexamethasone (VAD) that may be used to treat patients of MM with renal insufficiency. The VAD regimen (Barlogie et al. 1984) has been shown to produce rapid rate of response, but the survival duration achieved is no longer than the most commonly used standard treatment combination of melphalan and prednisolone (Alexanian et al. 1990). It has been shown that the activity of the VAD regimen is almost entirely due to high-dose dexamethasone (Alexanian et al. 1992). Probably only mature plasma cells are killed by dexamethasone, whereas the immature, clonogenic myeloma compartment can be rescued from dexamethasoneinduced apoptosis by IL-6, an important cytokine secreted by myeloma cells.

The future use of GNP conjugation with VS and perhaps, the other MM drugs, may open up possibilities for newer chemotherapeutic regime in which this dual role of the nanoparticle is exploited, lower drug concentration may be used and higher drug potential may be achieved at a much reduced level of toxicity.
Acknowledgement We thank DST, India (grant no. SR/NM/NS-28 2007) for supporting the research.

\section{References}

Alexanian R, Barlogie B et al (1990) VAD-based regimens as primary treatment for multiple myeloma. Am J Hematol 33 (2):86-89

Alexanian R, Dimopoulos MA et al (1992) Primary dexamethasone treatment of multiple myeloma. Blood 80(4):887-890

Anselmi C, Ettorre A et al (2002) In vitro induction of apoptosis vs. necrosis by widely used preservatives: 2-phenoxyethanol, a mixture of isothiazolinones, imidazolidinyl urea and 1, 2pentanediol. Biochem Pharmacol 63(3):437-453

Barbu E, Molnar E et al (2009) The potential for nanoparticle-based drug delivery to the brain: overcoming the blood-brain barrier. Expert Opin Drug Deliv 6(6):553-565

Barlogie B, Smith L et al (1984) Effective treatment of advanced multiple myeloma refractory to alkylating agents. N Engl J Med 310(21):1353-1356

Calatozzolo C, Gelati M et al (2005) Expression of drug resistance proteins Pgp, MRP1, MRP3, MRP5 and GST-pi in human glioma. J Neurooncol 74(2):113-121

Catelas I, Petit A et al (2005) Quantitative analysis of macrophage apoptosis vs. necrosis induced by cobalt and chromium ions in vitro. Biomaterials 26(15):2441-2453

Chompoosor A, Saha K et al (2010) The role of surface functionality on acute cytotoxicity, ROS generation and DNA damage by cationic gold nanoparticles. Small 6 (20):2246-2249 
Daniel MC, Tsvetkova IB et al (2010) Role of surface charge density in nanoparticle-templated assembly of bromovirus protein cages. ACS Nano 4(7):3853-3860

de Carcer G, Perez de Castro I et al (2007) Targeting cell cycle kinases for cancer therapy. Curr Med Chem 14(9):969-985

Dickson MA, Schwartz GK (2009) Development of cell-cycle inhibitors for cancer therapy. Curr Oncol 16(2):36-43

Dvorak C (2006) Common complaints, difficult diagnosis: multiple myeloma. J Am Acad Nurse Pract 18(5):190-194

Huet S, Marie JP et al (1998) Reference method for detection of Pgp mediated multidrug resistance in human hematological malignancies: a method validated by the laboratories of the French Drug Resistance Network. Cytometry 34(6):248-256

Johansson M, Persson JL (2008) Cancer therapy: targeting cell cycle regulators. Anticancer Agents Med Chem 8(7):723-731

Kekre N, Griffin C et al (2005) Pancratistatin causes early activation of caspase- 3 and the flipping of phosphatidyl serine followed by rapid apoptosis specifically in human lymphoma cells. Cancer Chemother Pharmacol 56(1):29-38

Kimling J, Maier $\mathrm{M}$ et al (2006) Turkevich method for gold nanoparticle synthesis revisited. J Phys Chem B 110 (32):15700-15707

Kostrzewa RM (2000) Review of apoptosis vs. necrosis of substantia nigra pars compacta in Parkinson's disease. Neurotox Res 2(23):239-250

Lapotko DO, Lukianova E et al (2006) Selective laser nanothermolysis of human leukemia cells with microbubbles generated around clusters of gold nanoparticles. Lasers Surg Med 38 (6):631-642

Legrand O, Simonin G et al (1999) Both Pgp and MRP1 activities using calcein-AM contribute to drug resistance in AML. Adv Exp Med Biol 457:161-175

Lieberthal W, Triaca V et al (1996) Mechanisms of death induced by cisplatin in proximal tubular epithelial cells: apoptosis vs. necrosis. Am J Physiol 270(4 Pt 2):F700-F708
Ma P, Dong X et al (2009) Development of idarubicin and doxorubicin solid lipid nanoparticles to overcome Pgp-mediated multiple drug resistance in leukemia. J Biomed Nanotechnol 5(2):151-161

Murphy EA, Majeti BK et al (2008) Nanoparticle-mediated drug delivery to tumor vasculature suppresses metastasis. Proc Natl Acad Sci USA 105(27):9343-9348

Patra HK, Banerjee S et al (2007) Cell selective response to gold nanoparticles. Nanomedicine 3(2):111-119

Rauch C, Pluen A (2007) Multi drug resistance-dependent "vacuum cleaner" functionality potentially driven by the interactions between endocytosis, drug size and Pgp-like transporters surface density. Eur Biophys J 36(2):121-131

Raucher D, Moktan S et al (2009) Therapeutic peptides for cancer therapy. Part II - cell cycle inhibitory peptides and apoptosisinducing peptides. Expert Opin Drug Deliv 6(10):1049-1064

Sanchez P, Llorente MT et al (2000) Flow cytometric detection of micronuclei and cell cycle alterations in fish-derived cells after exposure to three model genotoxic agents: mitomycin $\mathrm{C}$, vincristine sulfate and benzo(a)pyrene. Mutat Res 465(1-2):113-122

Vairetti M, Ferrigno A et al (2005) Apoptosis vs. necrosis: glutathione-mediated cell death during rewarming of rat hepatocytes. Biochim Biophys Acta 1740(3):367-374

Zhao F, Chen Y et al (2010a) Role of triptolide in cell proliferation, cell cycle arrest, apoptosis and histone methylation in multiple myeloma U266 cells. Eur J Pharmacol 646(1-3):1-11

Zhao F, Chen Y et al (2010b) Effects of triptolide on RIZ1 expression, proliferation, and apoptosis in multiple myeloma U266 cells. Acta Pharmacol Sin 31(6):733-740

Zhao Y, Trewyn BG et al (2009) Mesoporous silica nanoparticle-based double drug delivery system for glucose-responsive controlled release of insulin and cyclic AMP. J Am Chem Soc 131 (24):8398-8400

Zhu ZJ, Carboni R et al (2010) Surface properties dictate uptake, distribution, excretion, and toxicity of nanoparticles in fish. Small 6(20):2261-2265 